\title{
Clinical and Genetic Risk Factors Associated with Psoriatic Arthritis among Patients with Psoriasis
}

Di Yan · Richard Ahn $\cdot$ Stephen Leslie $\cdot$ Wilson Liao

Received: July 23, 2018 / Published online: October 20, 2018

(c) The Author(s) 2018

\begin{abstract}
Introduction: Psoriatic arthritis (PsA) is a chronic, inflammatory arthritis that affects an estimated $30 \%$ of patients with psoriasis. PsA is underdiagnosed in primary care and dermatology clinics due to a variety of reasons, including failure of healthcare providers to ask about symptoms, overlap of symptoms and signs with other rheumatologic conditions, and lack of a specific diagnostic test. A delay in PsA diagnosis and treatment, even as short as 6 months, can lead to decreased quality of life, increased joint
\end{abstract}

Enhanced digital features To view enhanced digital features for this article go to https://doi.org/10.6084/ m9.figshare.7151903.

Electronic supplementary material The online version of this article (https://doi.org/10.1007/s13555018-0266-x) contains supplementary material, which is available to authorized users.

D. Yan · W. Liao $(\bowtie)$

Department of Dermatology, University of California San Francisco, San Francisco, CA, USA e-mail: wilson.liao@ucsf.edu

R. Ahn

Department of Microbiology, Immunology and Molecular Genetics, Institute for Quantitative and Computational Biosciences, University of California, Los Angeles, CA, USA

S. Leslie

Centre for Systems Genomics, Schools of Mathematics and Statistics, and BioSciences, The University of Melbourne, Melbourne, VIC, Australia damage, and worse long-term physical function. In this study, we sought to identify the clinical and genetic factors that help discriminate patients with PsA from those with cutaneous psoriasis only.

Methods: We analyzed a cohort of 974 psoriasis patients at an academic medical center, of whom 175 had confirmed PsA, and performed univariate, multivariate, and predictive modeling to determine factors associated with PsA.

Results: The univariate analysis revealed significant positive associations of PsA with age, nail involvement, scalp involvement, skin fold involvement, elbow/knee involvement, psoriasis severity, plaque subtype, erythrodermic subtype, hypertension, type 2 diabetes, and coronary artery disease, and a significant negative association of PsA with the human leukocyte antigen (HLA)-C*06:02 allele. In the multivariate analysis, nail involvement, type 2 diabetes, and pustular psoriasis remained significantly associated with PsA, while HLA-C*06:02 positivity remained protective. There was a trend towards an association of PsA with older age, younger age of psoriasis onset, and skin fold involvement, while there was protective trend for smoking. A predictive model including both clinical and genetic factors showed reasonable discriminative ability between psoriasis and PsA, with an area under the curve of 0.87 for a receiver operating characteristic curve.

Conclusion: This study identified a number of clinical and genetic features that could help stratify patients who are at higher risk for 
having PsA and for whom rheumatology referral may be beneficial.

Keywords: Association;

Demographics; Genetics; Prediction; Psoriasis; Psoriatic arthritis; Risk

\section{INTRODUCTION}

Psoriatic arthritis (PsA) is a chronic, inflammatory arthritis that affects an estimated $30 \%$ of patients with psoriasis [1]. Patients with PsA have a higher prevalence of cardio-metabolic comorbidities [2]. They are also at risk for permanent deformity and disability, decreased quality of life (QoL), reduced work productivity, increased risk of death, and higher healthcare utilization [3]. Currently, more than $50 \%$ of patients with PsA are untreated or undertreated with only topical medications [4]. Delays in PsA treatment, even as short as 6 months, can lead to decreased QoL, increased joint damage, and worse long-term physical function [5]. Early recognition of PsA is therefore crucial to reducing joint destruction and improving long-term disease outcomes.

While studies have found associations between PsA, smoking [6-8], obesity [9], genetic risk factors [10], and various psoriasis subtypes $[11,12]$, these studies looked at a limited set of variables and the results are sometimes contradictory or have yet to be confirmed by additional research. Furthermore, to our knowledge, no studies have developed models to predict the risk or presence if PsA.

The aim of this study was to assess the association of PsA with a multidimensional panel of clinical, demographic, environmental, and genetic factors and to develop clinically useful statistical models that help identify patients who are likely to have PsA.

\section{METHODS}

\section{Patient Population}

Cross-sectional survey data were collected from 974 psoriasis patients seen at the University of California, San Francisco Department of
Dermatology between 2006 and 2017. Subjects included both treatment-naïve patients and those on topical, systemic, and phototherapy treatments for psoriasis. Study subjects completed a detailed survey, providing information on demographic characteristics, personal and family medical history, smoking history, age of onset, history of previous and current psoriasis treatments, and clinical features (subtype, affected areas, severity [defined by percentage body surface area covered], aggravating factors, presence of joint symptoms) of psoriasis. The diagnosis of psoriasis was confirmed by a boardcertified dermatologist who had examined the patient prior to enrollment. A confirmed diagnosis of PsA was defined as a diagnosis of PsA made by a rheumatologist or dermatologist with patient report of joint symptoms. Possible PsA was defined as the absence of an official diagnosis by a physician in the presence of current symptoms of joint swelling, pain, and stiffness in one or more joints. The absence of PsA was defined as a lack of clinical signs and symptoms as assessed by the physician, no patient-reported prior diagnosis of PsA by a physician, and no patient reported symptoms of possible PsA. For this study, only subjects with confirmed PsA were classified as having PsA and compared to subjects without PsA, while those with possible PsA were omitted from the analysis. This study was approved by the University of California, San Francisco's institutional review board and informed consent was obtained from all subjects enrolled in the study.

\section{Genetic Analysis}

DNA was extracted from whole blood or saliva samples donated by the study participants using the Qiagen DNeasy kit (QIAGEN, Hilden, Germany) or the Oragene prepIT DNA extraction kit (DNA Genotek, Ottawa, ON, Canada), respectively. The DNA samples were genotyped using the Affymetix UK Biobank Array (Affymetix Inc., Santa Clara, CA, USA). The copy number of human leukocyte antigen (HLA)$\mathrm{C}^{\star}$ 06:02 and HLA-B*27 alleles were imputed from array data using HLA*IMP2, which has an accuracy of $\geq 95 \%$ for HLA-C and HLA-B [13]. 


\section{Statistical Analyses}

All statistical analyses were performed in $\mathrm{R}$ (version 3.3.2; R Foundation for Statistical Computing, Vienna, Austria). The initial pool of variables was selected based on evidence of possible associations with PsA in the literature and biological plausibility.

\section{Association Models}

To avoid over-fitting, the model was limited to one predictor for every ten events, with an event defined as the presence of PsA. Using this heuristic, we determined that our model could include a maximum of 17 predictor variables. For genetic variables we used a binary definition of positivity for the HLA-C*06:02 and HLA-B*27 alleles (patient did not have the HLA allele vs. the patient had at least one copy of the allele). Initial univariate analyses were conducted using Chi-squared tests or Fisher's exact tests for categorical variables and two-tailed $t$ tests for continuous variables. An a priori threshold of a $p$ value of $<0.25$ was used to select variables for inclusion in the subsequent multivariable logistic regression model. Demographic characteristics (age, age of onset, gender, body mass index $[\mathrm{BMI}]$ ) were forced into the model to control for potential confounders. A backward elimination method based on the $p$ value of the Wald test statistic was used to reduce the model to 17 predictor variables. Sensitivity analyses were conducted using alternative pre-defined $p$ value thresholds of 0.15 and 0.05 to determine whether a more stringent barrier to inclusion would alter the results. We also used an alternative definition of severity as a four-level ordinal variable.

\section{Prediction Models}

We constructed two multivariable logistic regression models to predict the presence of PsA. Model 1 included only factors that are known to predate the onset of PsA, such as age of psoriasis onset, gender, family history of psoriasis, and the copy number of HLA-C ${ }^{*} 06: 02$ and HLA-B27 alleles. The full data set was used to assess the diagnostic ability of model 1 and construct a receiver operating characteristic curve.

For model 2, which included variables that were associated with PsA and not necessarily predating PsA onset, the dataset was split into a training set (25\%) and independent test set used for validation $(75 \%)$. The training set was used to develop model 2 , while the test set was used to determine the internal validity of the model and construct the ROC curves. Stepwise regression based on the Akaike information criterion was used to build model 2 from a pool of all investigated variables. The variable, HLA$C^{\star} 06: 02$ positivity, was forced into model 2 as previous studies had suggested that this allele has a significant protective effect against the development of PsA [14-16]. The concordance statistic (c-index), defined as the area under the ROC curve was used to assess the predictive accuracy of the two predictive logistic regression models.

The presence of multicollinearity was assessed using the variance inflation factor. The generalized additive models approach was used to test the assumption that the continuous variables (age and age of onset) were linearly related to the logit in the logistic regression model [17]. The Hosmer-Lemeshow test was used to assess the models' goodness of fit.

\section{RESULTS}

\section{Description of Study Population}

The prevalence of confirmed PsA in our cohort was $18.0 \%(n=175)$, while the prevalence of subjects without confirmed PsA was 51\% $(n=497)$. The average age of our study population was 44.6 years; $55 \%$ of our cohort was male and $44.9 \%$ were female. The average BMI was $27.1 \mathrm{~kg} / \mathrm{m}^{2}$, a value which is considered to indicate clinically overweight and slightly above the national average [18]. Our study population drew from the Bay area of California and was predominantly Caucasian, Asian, and Hispanic/Latino. Characteristics of the training and internal validation cohorts are provided in 
Electronic Supplementary Material (ESM)

Table S1.

\section{Univariate Associations with PsA}

We first analyzed a number of demographic, clinical, and genetic variables on a univariate basis for association with PsA (Table 1). We found significant positive associations for age $(p=0.024)$, scalp psoriasis $(p=0.004)$, elbow/ knee psoriasis $(p=0.040)$, nail involvement $\left(p=8.2 \times 10^{-08}\right)$, skin fold involvement $(p=0.005)$, plaque subtype $(p=0.01)$, erythrodermic subtype $(p=0.014)$, severe psoriasis $\left(p=2.39 \times 10^{-5}\right), \quad$ hypertension $(p=0.003)$, type 2 diabetes $(p=0.024)$, and coronary artery disease $(p=0.045)$. We found a significant negative association for the HLA-C*06:02 allele $(p=0.003)$.

\section{Multivariate Association Model}

Next, we built a multivariate association model using a backward elimination method (Table 2). Among the demographic characteristics included in the final multivariable logistic regression model, only the age of psoriasis onset showed a trend towards a higher odds of having PsA $(p=0.050)$. For every 1 year increase in the age of onset, the odds of having PsA decreased by $3 \%$.

Clinical features, such as lesion location and psoriasis subtype, were also associated with increased odds of PsA. Patients who had nail psoriasis had a 3.01-fold higher odds of having PsA (95\% confidence interval [CI] 1.39-6.66; $p=0.006)$ than did individuals without nail involvement. Those with pustular psoriasis had a 6.11-fold odds of having PsA (95\% CI $1.40-36.41 ; p=0.022)$ than did those who did not.

In terms of co-morbidities, subjects who had type 2 diabetes had a significant 5.27-fold increase in the odds of having PsA (95\% CI $1.17-36.8 ; p=0.037)$ that was independent of current BMI and hypertension status.

Genetically, individuals who were positive (heterozygous or homozygous) for the HLA$C^{\star} 06: 02$ allele had a $67 \%$ reduction in the odds of having PsA relative to patients who lacked HLA- $C^{*} 06: 02$. There was no significant difference in the odds of PsA for patients who had the HLA-B*27 allele.

A number of other variables showed a trend toward association with PsA in the multivariate model, but these were not statistically significant. These included intertriginous psoriasis, plaque psoriasis, severe psoriasis, and smoking (Table 2).

\section{Sensitivity Analysis}

Reducing the $p$ value for entry into the model to 0.15 or 0.05 did not significantly change the parameter estimates or $p$ values. The only variable appreciably affected was pustular psoriasis status, which had a $p$ value of 0.064 in the univariate analyses and therefore did not make the cutoff for inclusion in the model that used an entry criteria of $p<0.05$. The results of these sensitivity analyses (ESM Tables S2, S3, S4) suggest that our association model is fairly robust. Moreover, we conducted an additional sensitivity analysis examining whether forcing the variable of ethnicity/race (Caucasian, Asian, and Hispanic/Latino, Other) into the models altered the results and found that the none of our findings was significantly altered (ESM Appendix).

\section{Multivariate Predictive Models}

The association model assessed the relationship between PsA and the most significant factors found on univariate analysis while controlling for demographic variables; in comparison, the predictive models evaluated how well a set of variables predict the presence of PsA. The results of the multivariable logistic regression model using only factors that are known to predate PsA onset (model 1) are shown in Table 3. Only the HLA-C*06:02 allele was found to be a statistically significant predictor of PsA status $(p=0.001)$. Patients with one or more copies of the HLA-C*06:02 allele had a 55\% reduction in the odds of having PsA compared to individuals who did not have any copies of the HLA$\mathrm{C}^{\star}$ 06:02 allele. The c-index was 0.615 (Fig. 1, 
Table 1 Univariate analysis of demographic, clinical, and genetics features associated with psoriatic arthritis

\begin{tabular}{|c|c|c|c|c|}
\hline \multirow{2}{*}{$\begin{array}{l}\text { Demographic, clinical, and } \\
\text { genetics features }\end{array}$} & \multicolumn{2}{|l|}{ Patient group } & \multirow[t]{2}{*}{$p$ value $^{\mathrm{a}}$} & \multirow[t]{2}{*}{ OR $[95 \% \mathrm{CI}]^{\mathrm{b}}$} \\
\hline & PsA & No PsA & & \\
\hline Mean age (years) & 48.46 & 45.06 & $0.024^{*}$ & $0.002[0.0003-0.004]$ \\
\hline Age of psoriasis onset (years) & 26.07 & 28.09 & 0.193 & $\begin{array}{l}-0.001[-0.003 \text { to } \\
0.0006]\end{array}$ \\
\hline Gender, $n$ female/total ( $\%$ female $)$ & $85 / 175(48.6 \%)$ & $\begin{array}{l}208 / 495 \\
(42.0 \%)\end{array}$ & 0.158 & $1.30[0.92-1.84]$ \\
\hline BMI & 27.84 & 26.63 & $0.094^{\mathrm{e}}$ & $0.006[-0.001$ to 0.014$]$ \\
\hline $\begin{array}{l}\text { Family history of psoriasis, } n \\
\text { yes/total }\end{array}$ & $98 / 175(56.0 \%)$ & $\begin{array}{l}254 / 488 \\
\quad(52.0 \%)\end{array}$ & 0.418 & $1.17[0.83-1.66]$ \\
\hline \multicolumn{5}{|l|}{ (\% yes) } \\
\hline HLA-C*06:02 positivity & $38 / 175(21.7 \%)$ & $\begin{array}{l}156 / 497 \\
(31.4 \%)\end{array}$ & $0.003^{* *}$ & $0.53[0.35-0.80]$ \\
\hline HLA-B*27 positivity & $11 / 175(6.3 \%)$ & $28 / 497(5.6 \%)$ & 1.000 & $1.05[0.51-2.16]$ \\
\hline Smoking, $n$ yes/total ( $\%$ yes) & $6 / 175(3.4 \%)$ & $30 / 472(6.4 \%)$ & 0.211 & $0.52[0.21-1.28]$ \\
\hline \multicolumn{5}{|l|}{ Clinical factors } \\
\hline Scalp involvement & $\begin{array}{l}153 / 175 \\
(87.4 \%)\end{array}$ & $\begin{array}{l}382 / 497 \\
(76.9 \%)\end{array}$ & $0.004^{* *}$ & $2.09[1.28-3.43]$ \\
\hline Ear involvement & $\begin{array}{l}114 / 175 \\
(65.1 \%)\end{array}$ & $\begin{array}{l}283 / 497 \\
(56.9 \%)\end{array}$ & $0.078^{\mathrm{e}}$ & $1.40[0.98-2.01]$ \\
\hline Elbow/knee involvement & $\begin{array}{l}145 / 175 \\
(82.9 \%)\end{array}$ & $\begin{array}{l}371 / 497 \\
(74.6 \%)\end{array}$ & $0.040^{*}$ & $1.62[1.04-2.53]$ \\
\hline Arm/leg involvement & $\begin{array}{l}152 / 175 \\
(86.9 \%)\end{array}$ & $\begin{array}{l}423 / 497 \\
(85.1 \%)\end{array}$ & 0.660 & $1.16[0.70-1.91]$ \\
\hline Trunk involvement & $\begin{array}{l}133 / 175 \\
(76.0 \%)\end{array}$ & $\begin{array}{l}346 / 497 \\
(69.6 \%)\end{array}$ & 0.132 & $1.38[0.93-2.05]$ \\
\hline Face involvement & $79 / 175(45.1 \%)$ & $\begin{array}{l}206 / 497 \\
(41.4 \%)\end{array}$ & 0.446 & $1.16[0.82-1.64]$ \\
\hline Genital involvement & $63 / 175(36.0 \%)$ & $\begin{array}{l}142 / 497 \\
(28.6 \%)\end{array}$ & $0.082^{\mathrm{e}}$ & $1.41[0.98-2.03]$ \\
\hline Nail involvement & $\begin{array}{l}101 / 175 \\
(57.7 \%)\end{array}$ & $\begin{array}{l}170 / 497 \\
(34.2 \%)\end{array}$ & $8.21 \times 10^{-08_{* * *}}$ & $2.63[1.85-3.74]$ \\
\hline Palm involvement & $40 / 175(22.9 \%)$ & $\begin{array}{l}103 / 497 \\
(20.7 \%)\end{array}$ & 0.627 & $1.13[0.75-1.71]$ \\
\hline Sole involvement & $39 / 175(22.3 \%)$ & $83 / 497(16.7 \%)$ & 0.125 & $1.43[0.93-2.19]$ \\
\hline Skin fold involvement & $79 / 175(45.1 \%)$ & $\begin{array}{r}162 / 497 \\
(32.6 \%)\end{array}$ & $0.005^{* *}$ & $1.69[1.19-2.40]$ \\
\hline
\end{tabular}


Table 1 continued

\begin{tabular}{|c|c|c|c|c|}
\hline \multirow{2}{*}{$\begin{array}{l}\text { Demographic, clinical, and } \\
\text { genetics features }\end{array}$} & \multicolumn{2}{|l|}{ Patient group } & \multirow[t]{2}{*}{$p$ value $^{\mathrm{a}}$} & \multirow[t]{2}{*}{ OR $[95 \% \mathrm{CI}]^{\mathrm{b}}$} \\
\hline & PsA & No PsA & & \\
\hline Gluteal cleft involvement & $27 / 175(15.4 \%)$ & $84 / 497(16.9 \%)$ & 0.739 & $0.90[0.56-1.44]$ \\
\hline Plaque subtype & $\begin{array}{l}157 / 175 \\
(89.7 \%)\end{array}$ & $\begin{array}{l}402 / 497 \\
(80.9 \%)\end{array}$ & $0.010^{*}$ & $2.06[1.21-3.52]$ \\
\hline Guttate subtype & $52 / 175(29.7 \%)$ & $\begin{array}{l}137 / 497 \\
(27.6 \%)\end{array}$ & 0.656 & $1.11[0.76-1.62]$ \\
\hline Pustular subtype & $11 / 175(6.3 \%)$ & $14 / 497(2.8 \%)$ & $0.064^{\mathrm{e}}$ & $2.31[0.93-5.60]$ \\
\hline Erythrodermic subtype & $13 / 175(7.4 \%)$ & $14 / 497(2.8 \%)$ & $0.014^{*}$ & $2.77[1.27-6.01]$ \\
\hline Palmoplantar subtype & $13 / 175(7.4 \%)$ & $22 / 497(4.4 \%)$ & 0.181 & $1.73[0.85-3.52]$ \\
\hline Inverse subtype & $17 / 175(9.7 \%)$ & $29 / 497(5.8 \%)$ & 0.116 & $1.74[0.93-3.24]$ \\
\hline Severity & - & - & 0.0005 & - \\
\hline Mild & $6 / 169(3.6 \%)$ & $30 / 497(6.0 \%)$ & 0.262 & $0.55[0.23-1.35]$ \\
\hline Mild-moderate & $23 / 175(13.1 \%)$ & $\begin{array}{l}107 / 497 \\
(21.5 \%)\end{array}$ & $0.021^{*}$ & $0.55[0.34-0.90]$ \\
\hline Moderate-severe & $54 / 175(30.9 \%)$ & $\begin{array}{l}185 / 497 \\
(37.2 \%)\end{array}$ & 0.155 & $0.75[0.52-1.09]$ \\
\hline Severe ${ }^{c}$ & $88 / 175(50.3 \%)$ & $\begin{array}{l}159 / 497 \\
(32.0 \%)\end{array}$ & $2.39 \times 10^{-5_{* * *}}$ & $2.15[1.51-3.05]$ \\
\hline \multicolumn{5}{|l|}{ Comorbidities } \\
\hline Hypertension & $61 / 175(34.9 \%)$ & $\begin{array}{l}115 / 497 \\
(23.1 \%)\end{array}$ & $0.003^{* *}$ & $1.78[1.22-2.58]$ \\
\hline Diabetes, type 2 & $18 / 175(10.3 \%)$ & $25 / 497(5.0 \%)$ & $0.024^{*}$ & $2.16[1.15-4.07]$ \\
\hline Hypercholesterolemia $^{\mathrm{d}}$ & $38 / 175(21.7 \%)$ & $\begin{array}{l}119 / 497 \\
(23.9 \%)\end{array}$ & 0.6202 & $0.88[0.58-1.33]$ \\
\hline Hypertriglyceridemia $^{\mathrm{d}}$ & $16 / 175(9.1 \%)$ & $31 / 497(6.2 \%)$ & 0.261 & $1.51[0.81-2.84]$ \\
\hline Coronary artery disease & $12 / 175(6.8 \%)$ & $15 / 497(3.0 \%)$ & $0.045^{*}$ & $2.37[1.08-5.16]$ \\
\hline Stroke ${ }^{\mathrm{d}}$ & $4 / 175(2.3 \%)$ & $7 / 497(1.4 \%)$ & 0.660 & $1.64[0.35-6.53]$ \\
\hline
\end{tabular}

Values for variables are presented as the number of patients out of the total number of patients in that group, with the percentage of these patients given in parenthesis, with the exception of mean age and age of psoriasis onset $P s A$ Psoriatic arthritis, $O R$ odds ratio, $C I$ confidence interval, $B M I$ body mass index, $H L A$ human leukocyte antigen ${ }^{*},{ }^{* *},{ }^{* *}$ Results are statistically significant at ${ }^{*} p<0.05,{ }^{* *} p$ value $<0.01$, or highly significant at ${ }^{* * *} p<0.001$

${ }^{a} p$ values derived from $t$-tests or Wilcoxon rank sum tests for continuous variables and Chi squared or Fisher's exact tests for categorical variables

b ORs for continuous variables (age, age of onset, BMI) are per unit increase

c $P$ value for severity included in model as severe or not severe (binary)

d Variables with $p>0.25$

e Indicates a trend towards statistical significance $(p$ value $<0.1)$ 
suggesting that this limited model was only slightly better than random prediction (c-index of 0.500 ).

The results of model 2, derived from stepwise regression and using independent training and validation datasets are shown in Table 4 . The c-index for model 2 in the training and test sets were 0.820 and 0.874 , respectively (Fig. 2), which are significantly higher than that for model 1. Age of psoriasis onset, nail involvement, type 2 diabetes, and hypertriglyceridemia were all significant predictors of PsA in the derivation cohort. The Hosmer-Lemeshow test was not significant for any of the logistic regression models (model 2: $p=0.978$ ), suggesting that the predicted and observed values were well calibrated with one another.

\section{DISCUSSION}

Our multifactorial analysis of demographic, clinical, environmental, and genetic risk factors of PsA revealed new characteristics associated with PsA and demonstrate a novel predictive model that can help distinguish between those who have PsA and those who do not. For example, in one previous study the HLA-

Table 2 Multivariable logistic regression model of psoriatic arthritis

\begin{tabular}{lll}
\hline Variable $^{\mathbf{a}}$ & Odds ratio [95\% CI $]^{\mathbf{b}}$ & $\boldsymbol{p}_{\text {value }}$ \\
\hline Intercept & $0.05[0.00-0.53]$ & $0.015^{*}$ \\
Nail involvement & $3.01[1.39-6.66]$ & $0.006^{* *}$ \\
HLA-C $06: 02$ positivity & $0.33[0.13-0.77]$ & $0.013^{*}$ \\
Diabetes, type 2 & $5.27[1.21-24.5]$ & $0.029^{*}$ \\
Pustular subtype & $6.11[1.17-36.8]$ & $0.037^{*}$ \\
Age of psoriasis onset & $0.97[0.94-1.00]$ & $0.050^{\mathbf{c}}$ \\
Smoking & $0.29[0.06-1.01]$ & $0.076^{\mathbf{c}}$ \\
Skin fold involvement & $2.09[0.91-4.89]$ & $0.085^{\mathbf{c}}$ \\
Age & $1.03[1.00-1.06]$ & $0.091^{\mathbf{c}}$ \\
Severe psoriasis & $1.92[0.88-4.21]$ & 0.101 \\
Soles involvement & $0.44[0.15-1.15]$ & 0.107 \\
Plaque subtype & $2.63[0.86-9.57]$ & 0.109 \\
Ears involvement & $0.54[0.22-1.29]$ & 0.166 \\
Elbows/knees involvement & $1.88[0.73-5.24]$ & 0.202 \\
Hypertension & $0.53[0.18-1.44]$ & 0.231 \\
Inverse subtype & $2.08[0.51-8.20]$ & 0.296 \\
Gender & $1.22[0.54-2.77]$ & 0.631 \\
BMI & $0.99[0.93-1.05]$ & 0.703 \\
\hline A back & & \\
\hline
\end{tabular}

A backward stepwise elimination procedure was used with a threshold of $p<0.25$ for inclusion of variables in the initial model

, ${ }^{* *}$ Results are statistically significant at ${ }^{*} p<0.05$ or ${ }^{* *} p$ value $<0.01$

a Variables listed in order of $p$ value

b ORs for continuous variables (age, age of onset, BMI) are per unit increase

${ }^{c} p$ value indicates a trend towards statistical significance $(p<0.1)$ 
Table 3 Results of a predictive multivariable logistic regression model for presence of psoriatic arthritis that includes only factors predating the onset of psoriatic arthritis (model 1)

\begin{tabular}{lll}
\hline Variable & Odds ratio $(\mathbf{9 5} \% \mathbf{C I})^{\mathbf{a}}$ & $\boldsymbol{p}$ value \\
\hline Intercept & $0.55[0.32-0.92]$ & $0.024^{*}$ \\
Age of psoriasis onset & $0.99[0.98-1.00]$ & $0.065^{\mathrm{c}}$ \\
Gender $^{\mathrm{b}}$ & $1.24[0.83-1.84]$ & 0.295 \\
HLA-C ${ }^{*} 06: 02$ positivity & $0.45[0.29-0.70]$ & $0.0005^{* * *}$ \\
HLA-B 27 positivity & $0.96[0.43-1.99]$ & 0.908 \\
Family history of psoriasis & $1.24[0.83-1.87]$ & 0.291 \\
\hline${ }^{*},{ }^{* *},{ }^{* *}$ Results are statistically significant at ${ }^{*} p<0.05,{ }^{* *} p$ value $<0.01$, or highly significant at ${ }^{* * *} p<0.001$ & \\
a ORs for continuous variables (age, age of onset, $\mathrm{BMI})$ are per unit increase & \\
b Odds ratio for females relative to the reference group of males & \\
${ }^{c}$ Indicates a trend towards statistical significance $(p<0.1)$ &
\end{tabular}

$C^{\star} 06: 02$ allele was associated with a tenfold increase in the risk of developing psoriasis [19], but the results of other studies suggest that the HLA-C*06:02 allele may be protective against the development of PsA. More specifically, in a cohort of 480 patients with PsA, Ho and colleagues showed that those with both the HLA$\mathrm{C}^{\star} 06: 02$ and HLA-DRB1*07 alleles had

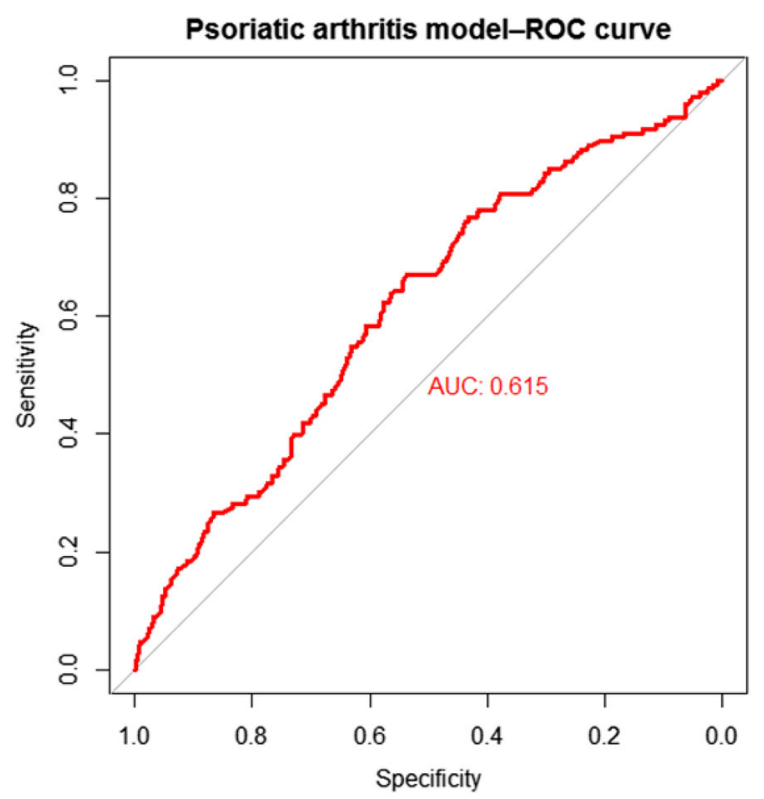

Fig. 1 Receiver operating characteristic (ROC) curve with area under the curve for model 1 significantly fewer damaged and involved joints than PsA patients who did not carry either allele [10]. Furthermore, Winchester and colleagues found that the frequency of the HLA-C*06:02 allele was lower in patients with psoriatic arthritis than in patients with only cutaneous psoriasis. In particular, Haroon and colleagues found that the HLA-C*06:02 allele was negatively associated with asymmetrical sacroiliitis. In our psoriasis cohort, which included patients with and without PsA, we found that positivity for the HLA-C*06:02 allele was negatively associated with PsA, although it should be noted that the frequency of the HLA-C*06:02 allele in both patients with PsA (21.7\%) and those with only psoriasis (31.4\%) was still higher than that in the general population.

Our study also identifies a new heightened association of cardio-metabolic disease with PsA as compared to psoriasis without PsA. In the univariate association analysis, the presence of hypertension, coronary artery disease, or type 2 diabetes was significantly associated with risk of PsA among those with psoriasis. Moreover, type 2 diabetes was strongly associated with PsA and was a significant predictor of PsA status in model 2. These results are consistent with a previous study by Coto-Segura and colleagues who have shown that PsA increases the risk of type 2 diabetes by 2.1 fold [20]. Interestingly, these authors found that the treatment of diabetes with glucagon-like peptide 1 (GLP-1) 
Table 4 Results of model 2, a multivariable logistic regression model for presence of psoriatic arthritis developed by stepwise regression from an initial pool of all demographic, clinical, environmental, and genetic factors

\begin{tabular}{lcc}
\hline Variable & Odds ratio $(\mathbf{9 5 \%} \mathbf{C I})$ & $\boldsymbol{p}$ value \\
\hline Model intercept & $0.11[0.01-0.87]$ & $0.045^{*}$ \\
Demographic characteristics & & $0.017^{*}$ \\
Age of psoriasis onset & $0.96[0.92-0.99]$ & $0.072^{\mathrm{b}}$ \\
Gender & $2.56[0.93-7.40]$ & \\
Heritable factors & & 0.327 \\
HLA-C*06:02 positivity & $0.60[0.20-1.64]$ & \\
Lesion location & & $0.067^{\mathrm{b}}$ \\
Ear involvement & $0.35[0.10-1.06]$ & $0.006^{* *}$ \\
Nail involvement & $3.92[1.5-10.8]$ & $0.084^{\mathrm{b}}$ \\
Comorbidities & & $0.025^{*}$ \\
Cardiovascular disease & $15.48[0.84-610]$ & $0.035^{*}$ \\
Diabetes, type 2 & $9.91[1.35-82.4]$ & \\
Hypertriglyceridemia & $0.03[0.00-0.48]$ & $0.082^{\mathrm{b}}$ \\
Subtype & & $0.051^{\mathrm{b}}$ \\
Plaque subtype & $4.25[0.94-26.4]$ & 0.157 \\
Inverse subtype & $5.26[1.01-23.0]$ & 0.154 \\
Pustular subtype & $4.77[0.57-47.4]$ & \\
Severity & $2.03[0.77-5.44]$ & \\
Severe psoriasis & &
\end{tabular}

${ }^{*},{ }^{* *}$ Results are statistically significant at ${ }^{*} p<0.05$ or ${ }^{* *} p$ value $<0.01$

a OR for females relative to the reference group of males

b Indicates a trend towards statistical significance $(p<0.1)$

analogues improved psoriasis symptoms in patients with both diabetes and psoriasis. Moreover, the authors of a prospective study conducted in the UK for 4 years involving 8124 adults with psoriasis and 76,599 adults without psoriasis found that psoriasis was a risk factor for incident diabetes [21]. Taken together, these results suggest a possible mechanistic link between PsA endocrine derangements in diabetes. While our study did not find a link between PsA and BMI or obesity, our survey data only included current BMI values and not the BMI values at age 18 years that ArabshahiSoltani and colleagues [9] found to be associated with PsA. It is possible that only childhood obesity and not obesity in later life is associated with PsA. In fact, PsA seems to be positively associated with a younger age of onset in our cohort as well as in other studies [9, 22], suggesting that the clinical course in a patient's childhood may set the stage for PsA in later life. Interestingly, hypertriglyceridemia was negatively associated with PsA in predictive model 2. High triglyceride levels may be a marker of treatment with systemic medications, such as retinoids [23], methotrexate [24], and tumor necrosis factor-alpha inhibitors [25-27]; these medications have been associated with 
A Psoriatic arthritis model-ROC curve for training set

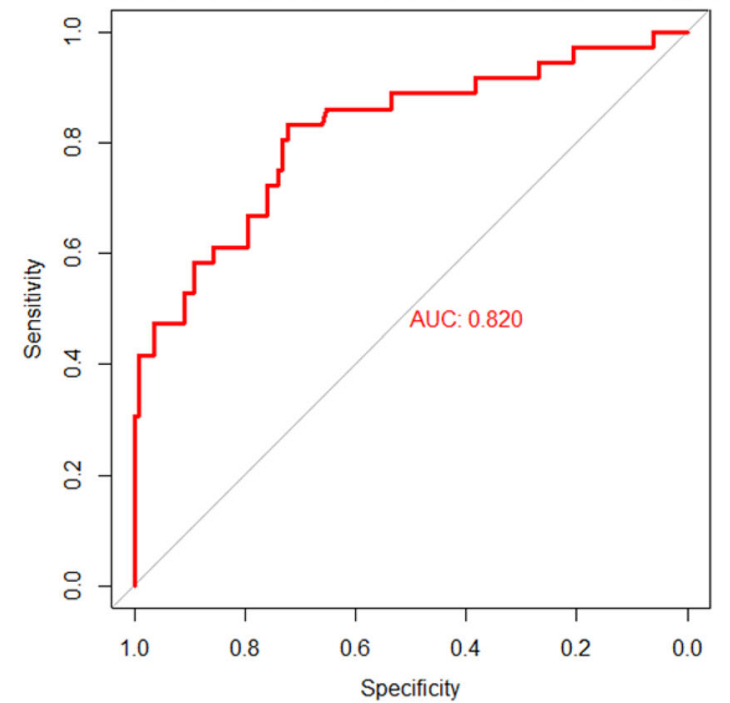

B

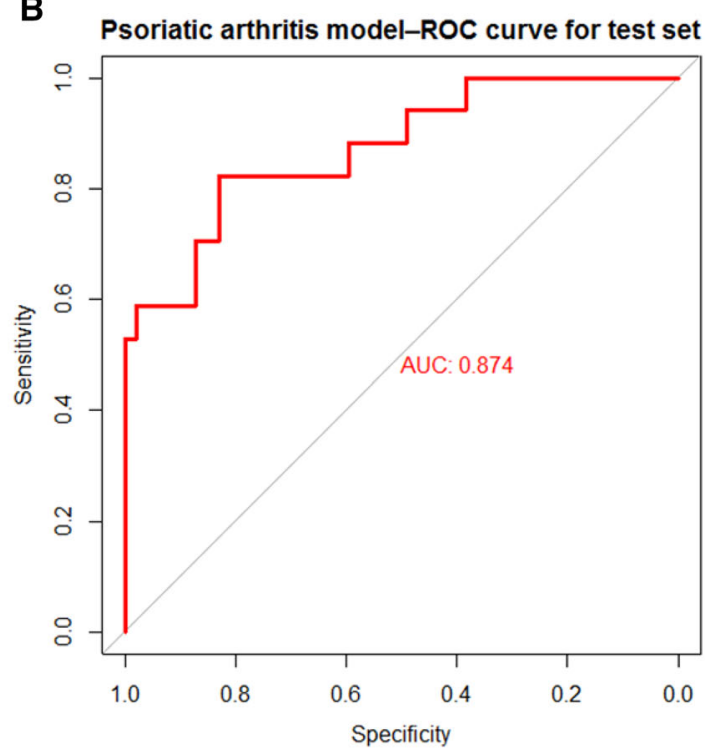

Fig. 2 ROC curves with area under the curve for model 2 in the training cohort (a) and test cohort $(\mathbf{b})$

increased triglyceride levels and may be used to treat or delay the onset of PsA.

Certain clinical features also seem to be important predictors of PsA status. For example, nail psoriasis is seen in both our study and those of others $[9,12]$ to be associated with PsA. "Localized" pustular psoriasis has also been associated with PsA in a Korean psoriasis cohort studied by Choi and colleagues, although their study methods did not specify details on the covariates used [22]. Our models demonstrate an association between pustular psoriasis and PsA. The association between PsA and a more extensive form of pustular psoriasis is supported by the study of David and colleagues who described four cases of generalized pustular psoriasis occurring with systemic amyloidosis, PsA, and nephrotic syndrome [28]. Overall, more severe and extensive psoriasis has been positively linked to PsA $[9,12,22]$ and is a significant predictor of PsA in model 2.

Some results of our study do differ from those reported in the literature. For example, while Wilson et al. described an association between PsA and inverse psoriasis [12], this was not seen in our cohort. However, involvement of the skin folds did trend towards a higher odds of having PsA. It is possible that the rarity of inverse psoriasis in our cohort $(7.8 \%$ overall; 9.7\% in PsA-positive subjects and 5.8\% in PsAnegative subjects) may have limited our power to identify associations with PsA status. Wilson et al. [12] also reported a link between scalp psoriasis and PsA [12]. In our study, scalp psoriasis was significantly different in the univariate Chi-squared analysis, but it was not significantly associated with PsA in the multivariate logistic regression analysis nor was it a significant predictor of PsA status in model 2. This discrepancy may be due, in part, to differences in the statistical models used. Also, while the multivariable Cox proportional hazards models used by Wilson et al. [12] adjusted only for age, sex, and calendar year, the logistic regression models used in our study controlled for numerous other clinical and genetic factors. Thus, it is possible that there was no significant association between our models because they controlled for additional confounders that may have driven the relationship between scalp psoriasis and PsA in the analysis performed by Wilson et al. [12]. 
It is important to note that our study is limited by the retrospective and cross-sectional nature of our data, which prevented us from distinguishing the directionality of the relationship between PsA and the risk factors explored in our models. Furthermore, the cross-sectional data also prevented us from estimating the future risk of PsA, although our models can be used to help clinicians in stratifying patients who are more likely to have PsA and those who are not. Finally, the lack of precise characterization of joint involvement hampered full interpretation of study.

\section{CONCLUSIONS}

In this study we evaluated the association of PsA with a wide variety of risk factors and developed predictive models that can help clinicians make earlier diagnoses of PsA and prevent the debilitating long-term sequelae of the disease. These models demonstrate the predictive value of HLA alleles in combination with clinical and demographic factors to identify "high suspicion" patients who may be candidates for additional work-up. Further prospective studies are needed to understand how these risk factors contribute to the development of PsA and whether they can be used to predict future disease onset.

\section{ACKNOWLEDGEMENTS}

Funding. This study was supported in part by grants to Wilson Liao (NIH R01AR065174, NIH U01AI119125) and Di Yan (National Psoriasis Foundation Fellowship). No funding was received for article processing charges.

Authorship. All named authors meet the International Committee of Medical Journal Editors (ICMJE) criteria for authorship for this article, take responsibility for the integrity of the work as a whole, and have given their approval for this version to be published.

Disclosures. Di Yan, Richard Ahn, Stephen Leslie, and Wilson Liao have nothing to disclose.
Compliance with Ethics Guidelines. All procedures performed in studies involving human participants were in accordance with the ethical standards of the institutional and/or national research committee and with the 1964 Helsinki declaration and its later amendments or comparable ethical standards. Informed consent was obtained from all individual participants included in the study.

Data Availability. The datasets during and/ or analyzed during the current study are available from the corresponding author on reasonable request.

Open Access. This article is distributed under the terms of the Creative Commons Attribution-NonCommercial 4.0 International License (http://creativecommons.org/licenses/ by-nc/4.0/), which permits any noncommercial use, distribution, and reproduction in any medium, provided you give appropriate credit to the original author(s) and the source, provide a link to the Creative Commons license, and indicate if changes were made.

\section{REFERENCES}

1. Ritchlin CT, Colbert RA, Gladman DD. Psoriatic arthritis. N Engl J Med. 2017;376(10):957-70.

2. Girolomoni G, Gisondi P. Psoriasis and systemic inflammation: underdiagnosed enthesopathy. J Eur Acad Dermatol Venereol. 2009;23[Suppl 1]:3-8.

3. Betteridge N, Boehncke WH, Bundy C, Gossec L, Gratacos J, Augustin M. Promoting patient-centred care in psoriatic arthritis: a multidisciplinary European perspective on improving the patient experience. J Eur Acad Dermatol Venereol. 2016;30(4):576-85.

4. Kavanaugh A, Helliwell P, Ritchlin CT. Psoriatic arthritis and burden of disease: patient perspectives from the population-based multinational assessment of psoriasis and psoriatic arthritis (MAPP) survey. Rheumatol Ther. 2016;3(1):91-102.

5. Haroon M, Gallagher P, FitzGerald O. Diagnostic delay of more than 6 months contributes to poor radiographic and functional outcome in psoriatic arthritis. Ann Rheum Dis. 2015;74(6):1045-50. 
6. Duffin KC, Freeny IC, Schrodi SJ, et al. Association between IL13 polymorphisms and psoriatic arthritis is modified by smoking. J Invest Dermatol. 2009;129(12):2777-83.

7. Li W, Han J, Qureshi AA. Smoking and risk of incident psoriatic arthritis in US women. Ann Rheum Dis. 2012;71(6):804-8.

8. Eder L, Shanmugarajah S, Thavaneswaran A, et al. The association between smoking and the development of psoriatic arthritis among psoriasis patients. Ann Rheum Dis. 2012;71(2):219-24.

9. Soltani-Arabshahi R, Wong B, Feng BJ, Goldgar DE, Duffin KC, Krueger GG. Obesity in early adulthood as a risk factor for psoriatic arthritis. Arch Dermatol. 2010;146(7):721-6.

10. Ho PY, Barton A, Worthington J, Thomson W, Silman AJ, Bruce IN. HLA-Cw6 and HLA-DRB1*07 together are associated with less severe joint disease in psoriatic arthritis. Ann Rheum Dis. 2007;66(6):807-11.

11. Ogdie A, Gelfand JM. Identification of risk factors for psoriatic arthritis: scientific opportunity meets clinical need. Arch Dermatol. 2010;146(7):785-8.

12. Wilson FC, Icen M, Crowson CS, McEvoy MT, Gabriel SE, Kremers HM. Incidence and clinical predictors of psoriatic arthritis in patients with psoriasis: a population-based study. Arthritis Rheum. 2009;61(2):233-9.

13. Dilthey A, Leslie S, Moutsianas L, et al. Multi-population classical HLA type imputation. PLoS Comput Biol. 2013;9(2):e1002877.

14. FitzGerald O, Haroon M, Giles JT, Winchester R. Concepts of pathogenesis in psoriatic arthritis: genotype determines clinical phenotype. Arthritis Res Ther. 2015;17:115.

15. Winchester R, Minevich G, Steshenko V, et al. HLA associations reveal genetic heterogeneity in psoriatic arthritis and in the psoriasis phenotype. Arthritis Rheum. 2012;64(4):1134-44.

16. Eder L, Chandran V, Pellet F, et al. Human leucocyte antigen risk alleles for psoriatic arthritis among patients with psoriasis. Ann Rheum Dis. 2012;71(1):50-5.

17. Generalized additive models. London: Chapman and Hall; 1990.

18. Prevention CfDCa. National Health and Nutrition Examination Survey: healthy weight, overweight, and obesity among U.S. adults; 2003. https://www. cdc.gov/nchs/data/nhanes/databriefs/adultweight. pdf

19. Gudjonsson JE, Karason A, Antonsdottir AA, et al. HLA-Cw6-positive and HLA-Cw6-negative patients with Psoriasis vulgaris have distinct clinical features. J Invest Dermatol. 2002;118(2):362-5.

20. Coto-Segura P, Eiris-Salvado N, Gonzalez-Lara L, et al. Psoriasis, psoriatic arthritis and type 2 diabetes mellitus: a systematic review and meta-analysis. Br J Dermatol. 2013;169(4):783-93.

21. Wan MT, Shin DB, Hubbard RA, Noe MH, Mehta NN, Gelfand JM. Psoriasis and the risk of diabetes: a prospective population-based cohort study. J Am Acad Dermatol. 2018;78(2):315 e1-322 e1.

22. Choi JW, Kim BR, Seo E, Youn SW. Could psoriatic arthritis be easily diagnosed from current suspicious physical findings in the dermatology clinic? Ann Dermatol. 2017;29(1):48-54.

23. Feingold KR, Grunfeld C. The effect of inflammation and infection on lipids and lipoproteins. In: De Groot LJ, Chrousos G, Dungan K, et al. (eds) Endotext. South Dartmouth: MDText.com, Inc.; 2000.

24. Navarro-Millan I, Charles-Schoeman C, Yang S, et al. Changes in lipoproteins associated with methotrexate or combination therapy in early rheumatoid arthritis: results from the treatment of early rheumatoid arthritis trial. Arthritis Rheum. 2013;65(6):1430-8.

25. Hassan S, Milman U, Feld J, et al. Effects of antiTNF-alpha treatment on lipid profile in rheumatic diseases: an analytical cohort study. Arthritis Res Ther. 2016;18(1):261.

26. Castro KR, Aikawa NE, Saad CG, et al. Infliximab induces increase in triglyceride levels in psoriatic arthritis patients. Clin Dev Immunol. 2011;2011:352686.

27. Saiki O, Takao R, Naruse Y, Kuhara M, Imai S, Uda $\mathrm{H}$. Infliximab but not methotrexate induces extrahigh levels of VLDL-triglyceride in patients with rheumatoid arthritis. J Rheumatol. 2007;34(10):1997-2004.

28. David M, Abraham D, Weinberger A, Feuerman EJ. Generalised pustular psoriasis, psoriatic arthritis and nephrotic syndrome associated with systemic amyloidosis. Dermatologica. 1982;165(3):168-71. 